\title{
Associations of moderate-to-vigorous physical activity with psychological problems and suicidality in Chinese high school students: A cross-sectional study
}

\author{
Mingli Liu ${ }^{\text {Corresp., } 1,2,3}{ }^{\text {, Jie Zhang }}{ }^{2,4}$, Kimberly E Kamper-DeMarco ${ }^{5}$, Elwin Hu ${ }^{6}$, Shuqiao Yao ${ }^{\text {Corresp. } 3}$ \\ ${ }^{1}$ Department of Psychology, Hunan University of Science and Technology, Xiangtan, Hunan, Country \\ 2 Department of Sociology, State University of New York Buffalo State, Buffalo, New York, USA \\ 3 Institute of Psychological Medicine, Second Xiangya Hospital, Central South University, Changsha, Hunan, China \\ 4 School of Sociology and Psychology, Central University of Finance and Economics, Beijing, Beijing, China \\ 5 Department of Psychology, State University of New York Buffalo State, Buffalo, New York, United States \\ 6 School of Psychology, Counselling and Psychotherapy, Cairnmillar Institute, Hawthorn East, Victoria, Australia \\ Corresponding Authors: Mingli Liu, Shuqiao Yao \\ Email address: liumingli@hnust.edu.cn, shuqiaoyao@csu.edu.cn
}

Background. The body of literature regarding the associations between physical activity and psychological problems lacks consensus. Moreover, the role of gender has been scarcely investigated. The present study sought to fill the gap in the data by examining the associations, if any, between moderate-to-vigorous physical activity (MVPA), psychological problems, and self-harm behaviors based on different biological genders (male - female).

Methods. A self-administered questionnaire was used to measure MVPA and multiple psychological problems, including depression and anxiety; general emotion, behavior, and social problems; Attention Deficit Hyperactivity Disorder(ADHD); Oppositional Defiant Disorder (ODD); conduct problems; and self-harm behaviors. Participants were a nationally representative sample of Chinese high school students $(N=13,349)$. A multiple logistic regression analysis of MVPA, stratified by gender, was conducted on the risk of psychological problems and self-harm behaviors in the total sample. Results. For boys, a high frequency of MVPA was associated with a lower risk of depression (OR $=0.68,95 \% \mathrm{Cl}$ : $0.57-0.81)$ and anxiety (OR $=0.71,95 \% \mathrm{Cl}: 0.53-0.96)$ compared with the reference group. The moderate frequency of MVPA was associated with a lower risk of ADHD (OR = 0.73, 95\% Cl: 0.56-0.94) compared with the reference group. For girls, both MVPA groups were associated with a lower risk of depression (moderate MVPA: OR $=0.81,95 \% \mathrm{Cl}$ : 0.70-0.94; high MVPA: OR $=0.67,95 \% \mathrm{Cl}: 0.54-0.83$ ) compared with the reference group. High MVPA was associated with a lower risk of ADHD (OR $=0.58,95 \% \mathrm{Cl}: 0.37-0.89$ ) compared with the reference group. Only moderate frequency of MVPA was associated with a lower risk of ODD (OR $=0.79,95 \% \mathrm{Cl}$ : 0.64-0.97). Conclusion. MVPA was 
associated with a lower risk of depression, anxiety, ADHD, and ODD in a gender-specific and MVPA frequency-specific manner. This implies that for specific psychological issues, PA interventions that are modified based on gender and frequency of MVPA may be more effective than PA interventions without these considerations. 


\section{Associations of moderate-to-vigorous physical activity with} 2 psychological problems and suicidality in Chinese high 3 school students: A cross-sectional study

Mingli Liu ${ }^{1,2,3}$, Jie Zhang ${ }^{2,4}$, Kimberly E. Kamper-DeMarco ${ }^{5}$, Elwin $\mathrm{Hu}^{6}$, Shuqiao Yao ${ }^{3}$ 6

${ }^{1}$ Department of Psychology, Hunan University of Science and Technology, Xiangtan, Hunan 411201, China

${ }^{2}$ Department of Sociology, State University of New York Buffalo State, New York 14222, USA

$11{ }^{3}$ Institute of Psychological Medicine, Second Xiangya Hospital, Central South University, 12 Changsha, Hunan 410011, China

$13{ }^{4}$ School of Sociology and Psychology, Central University of Finance and Economics, Beijing 14 100081, China

$15{ }^{5}$ Department of Psychology, State University of New York Buffalo State, New York 14222, 16 USA

$17{ }^{6}$ School of Psychology, Counselling and Psychotherapy, Cairnmillar Institute, Hawthorn East, 18 Victoria 3123, Australia.

Corresponding Author:

23 Shuqiao $\mathrm{Yao}^{1}$

24139 Middle Renmin Road, Changsha, Hunan, 410011, China

Mingli Liu ${ }^{2}$

2 Taoyuan Road, Xiangtan, Hunan, 411201, China 
36 Abstract

37 Background. The body of literature regarding the associations between physical activity and psychological problems lacks consensus. Moreover, the role of gender has been scarcely investigated. The present study sought to fill the gap in the data by examining the associations, if any, between moderate-to-vigorous physical activity (MVPA), psychological problems, and selfharm behaviors based on different biological genders (male - female).

Methods. A self-administered questionnaire was used to measure MVPA and multiple psychological problems, including depression and anxiety; general emotion, behavior, and social problems; Attention Deficit Hyperactivity Disorder (ADHD); Oppositional Defiant Disorder (ODD); conduct problems; and self-harm behaviors. Participants were a nationally representative sample of Chinese high school students $(\mathrm{N}=13,349)$. A multiple logistic regression analysis of MVPA, stratified by gender, was conducted on the risk of psychological problems and self-harm behaviors in the total sample.

Results. For boys, a high frequency of MVPA was associated with a lower risk of depression $(\mathrm{OR}=0.68,95 \% \mathrm{CI}: 0.57-0.81)$ and anxiety $(\mathrm{OR}=0.71,95 \% \mathrm{CI}: 0.53-0.96)$ compared with the reference group. The moderate frequency of MVPA was associated with a lower risk of ADHD $(\mathrm{OR}=0.73,95 \% \mathrm{CI}: 0.56-0.94)$ compared with the reference group. For girls, both MVPA groups were associated with a lower risk of depression (moderate MVPA: OR $=0.81,95 \% \mathrm{CI}$ : 0.70-0.94; high MVPA: OR =0.67, 95\% CI: 0.54-0.83) compared with the reference group. High MVPA was associated with a lower risk of ADHD (OR $=0.58,95 \%$ CI: 0.37-0.89) compared with the reference group. Only moderate frequency of MVPA was associated with a lower risk of ODD $(\mathrm{OR}=0.79,95 \% \mathrm{CI}$ : 0.64-0.97).

Conclusion. MVPA was associated with a lower risk of depression, anxiety, ADHD, and ODD in a gender-specific and MVPA frequency-specific manner. This implies that for specific psychological issues, PA interventions that are modified based on gender and frequency of MVPA may be more effective than PA interventions without these considerations.

3 Key words: Sex, Exercise, Depression, Anxiety, ADHD, Oppositional Defiant Disorder, 4 Conduct Problem, Suicidality

\section{Introduction}


It is well known in the literature that the occurrence of psychological problems rise sharply during adolescence (Gore et al., 2011; Patton et al., 2012). In fact, psychological problems are one of the leading causes of years of life lost in adolescents according to data from the World Health Organization's (WHO) Global Burden of Disease study (Gore et al., 2011; Mokdad et al., 2016). Furthermore, psychological problems during these years may influence health late in life (Sawyer et al., 2012). Therefore, psychological problems in adolescents are becoming a central public health concern (Carli et al., 2014; Merikangas et al., 2010). In order to create more appropriate prevention and intervention strategies, and subsequently decrease the burden of mental disorders, it is necessary to examine health risk factors for psychological problems in adolescents.

The literature has demonstrated that physical activity (PA) is not only strongly associated with physiological health benefits (Buffart et al., 2014), but is also associated with psychological health benefits, such as a reduction in depression and anxiety symptoms, increased self-esteem, social isolation, and even a decrease in suicidal ideation (Vancampfort et al., 2017; Werneck et al., 2019). For adolescents, it has been well documented that PA is significantly associated with a lower risk of depression (Annesi, 2005; Daley, Copeland et al., 2006). Furthermore, PA intervention trials have significantly reduced depression, anxiety, psychological distress, and emotional disturbance in adolescence (Ahn \& Fedewa, 2011; Brown et al., 2013). Although there is well established literature demonstrating the effects of PA on depression and anxiety, there are only a handful of studies reporting the associations of PA and other psychological problems, such as Attention Deficit Hyperactivity Disorder (ADHD; Gapin et al., 2011). However, the results from this body of literature remain inconsistent. For example, in one study, parent-rated ADHD was associated with low PA in adolescents with type 1 diabetes (Nylander et al., 2017). Recently, a study showed that increased moderate-to-vigorous PA (MVPA) was correlated with higher conduct problems in boys and hyperactivity in both genders (Ahn et al., 2018). It should be noted, however, that most of the studies mentioned above were highly heterogeneous regarding the participants and measurement instruments used (Larun et al., 2006). Therefore, further investigation is warranted and should be carried out in different population groups to determine whether associations exist between PA and adolescents' psychological problems. Furthermore, although PA seems to reduce depression and anxiety symptoms, little attention has been given to examining whether gender (i.e., male-female) influences psychological health 
98

outcomes. In a recent study (Wilkinson et al., 2017), anxiety related to body image was shown to be lower for American-born and Mexican-born females who engaged in more PA than for those who were less engaged in PA. However, this relationship was different for the males in the study. Specifically, the association was significant for Mexican-born males but not for American-born males. On the other hand, a negative correlation was found between the frequency of PA and anxiety for both boys and girls among European adolescents (McMahon et al., 2017). These results demonstrate that different cultures and/or countries may influence the relationship between PA and anxiety.

According to a detailed analysis of internationally comparable data, the lowest rates of PA are mostly observed among juveniles who live in low-income and middle-income countries or regions (Patton et al., 2012). However, research concerning the association between PA and adolescents' psychological problems in these areas, especially large-scale population-based investigations, is scarce compared to studies in developed countries or regions. In China, there have been several studies that have investigated the associations between PA and depression, anxiety, hostility/aggression, and suicide ideation in adolescents. A study conducted in Bengbu city, in southeastern China, found that junior high school students who performed vigorous PA (i.e., more than three days per week) were associated with fewer depressive symptoms but these results were not replicated with anxiety (Cao et al., 2011). However, another study based in the same city suggested that although low-to-moderate PA was associated with fewer depressive and psychotic symptoms, vigorous PA was associated with a higher risk of general psychological health issues, such as life satisfaction and self-esteem, hostility/aggression and suicide ideation (Tao et al., 2007). Additionally, a study conducted in Xuzhou, a city in eastern China, revealed that minimal PA was associated with depression and anxiety in junior high school students (Wang et al., 2014). Because the samples employed in the both studies mentioned above were limited to junior high school students based on their respective cities, located in eastern China, these results cannot be generalized to the broader Chinese population. Thus, studies with large nationally representative samples of Chinese participants are lacking. In attempt to remedy this shortcoming in the literature, one study was conducted across eight cities in China and revealed significant associations between PA (with moderate and vigorous PA measuresd separately) and reductions in depressive symptoms with pubertal stage examined as a moderator (Sun et al., 2014), however, this study neglected the potential confounding effect of other psychological 
129 problems and did not use recommended criteria for MVPA set by the WHO (WHO, 2010) for

130 child and adolescent psychological health.

131 The present study addressed some of these research gaps by analyzing the associations

132 between different volumes of MVPA and multiple psychological problems in both junior and

133 senior high school students across multiple cities in mainland China. These psychological

134 problems include depression; anxiety; general emotion, behavior, and social (GEBS) problems;

135 ADHD; ODD; conduct problems; and suicidal ideation/self-harm behavior (hereafter, self-harm

136 behaviors). Furthermore, given that gender has been proposed to moderate the relationship

137 between PA and some psychological problems (i.e., McMahon et al., 2017; Sun et al., 2014;

138 Wilkinson et al., 2017), we performed all analyses stratified by gender. We hypothesized that

139 higher MVPA would be more protective against psychological health in boys than in girls.

140 Materials \& Methods

\section{Participants}

142

High school pupils from $7^{\text {th }}$ to $12^{\text {th }}$ grade $(\mathrm{N}=13,349$; mean age: $15.18 \pm 1.90)$ from ten

143 urban areas (Beijing, Shanghai, Hangzhou, Guangzhou, Suzhou, Chengdu, Changsha, Shenyang,

144 Yinchuan, and Langfang) were recruited. These areas represent different areas across mainland

145 China. We used a stratified cluster sampling of all the public schools in these cities. First, we

146 categorized all schools in each city into three groups based on their level of academic

147 performance (low, medium, and high). Second, to make a representative sample of high school

148 students, two to three medium-level high schools in each city were randomly chosen (totaling 23

149 schools) to take part in the survey. Finally, one to three classes of students within these schools

150 were selected from each grade ( $7^{\text {th }}$ through $12^{\text {th }}$ grade) as the final sample of this study. More

151 detailed information on these study procedures can be found elsewhere (see Liu et al., 2016; Xin

152 et al., 2016). With the supervision of a research assistant, students completed the paper-based

153 questionnaires individually in class. All participants in this study and their parents provided

154 written informed consent. The Hunan Subjects Review Committee at Second Xiangya Hospital

155 of Central South University provided ethical approval (No: CSMC-2009S167).

\section{Measurements}


In this study, a self-administered questionnaire was used for the participants. It consisted

158

159

160

161

162

163

164

165

166

167

168

169

170

171

172

173

174

175

176

177

178

179

180

181

182

183

184

185

186

187

of three sections: sociodemographic information, including age, gender, grade, BMI, and subjective socioeconomic status (SES), the Youth Risk Behavior Survey and questionnaires assessing psychological problems and self-harm.

The Youth Risk Behavior Survey questionnaire (Brener et al., 2002) evaluated students' frequency of MVPA (days/week). An example question is "During the past seven days, on how many days were you physically active enough that your heart beat fast and you breathed hard for a total of $\geq 60$ minutes?" In order to adjust for the potential confounding role of screen-based sedentary behavior (Sun et al., 2014), we also examined participants' screen time (ST) on a typical school day (hours/day). ST includes watching television, playing video or computer games, or using the computer for noneducational purposes. Although the recommendation of MVPA is 7 days/week, the percentage of adolescents who meet this criterion is very low, which indicates that Chinese high school students generally lack MVPA. Based on the frequency of MVPA, the participants were categorized into three groups: no MVPA (0 days/week), moderate frequency of MVPA ( $\geq 1$ to $\leq 3$ days/week), and high frequency of MVPA ( $>3$ days/week, i.e., 4-7 days/week). In line with previous studies, ST was categorized into four groups: no ST (0 hours/day), low ST ( $\leq 1$ hours/day), moderate ST ( $>1$ to $<2$ hours/day), and high ST $(\geq 2$ hours/day) (Liu, Wu, \& Yao, 2016). SES was reported. Scores ranged from one to ten. Low scores (i.e., 1,2,3) represented low SES while high SES, was represented by high scores (i.e., $7,8,9)$.

A battery of questionnaires evaluated participants' psychological symptoms and selfharm behaviors. Depressive symptoms were examined using the Centers for Epidemiologic Studies-Depression Scale (CES-D, Radloff, 1991; Wang et al., 2013). The CES-D has 20 items, and responses range from 0 (rarely) to 3 (most of the time). Anxiety symptoms were measured using the Multidimensional Anxiety Scale for Children (MASC, March et al., 1997; Yao et al., 2007). The MASC has 39 items, and responses range from 0 (never applies to me) to 3 (often applies to me). GEBS problems, ADHD symptoms, ODD, and conduct problems were assessed with the Youth Self-Report (YSR; Achenbach, Dumenci, \& Rescorla, 2001; Yao et al., 2009). The YSR consists of 112 items divided into nine subscales with responses ranging from 0 (not true) to 2 (very true). Finally, self-harm behaviors were examined using a 5-item subscale from the Health-Risk Behavior Inventory for Chinese Adolescents (Wang et al., 2012). Responses

Peer) reviewing PDF | (2019:11:42937:2:0:NEW 6 Feb 2020) 
188 range from 0 (never) to 4 (always). For all these scales, high scores indicate higher levels of the 189 corresponding symptoms. Cronbach's alphas for all of the scales and subscales were good, 190 ranging from 0.71 to 0.95 .

191 Data was dichotomized based on the different cutoff scores (separated by gender) for 192 each psychological problem(i.e., depression (CES-D score $\geq 22$ for boys and $\geq 24$ for girls 193 (Primack et al., 2009; Roberts, Lewinsohn, \& Seeley, 1991); anxiety (T-score of MASC $\geq 65$;

194 March, 2007); GEBS problems (above the 90th percentile; Achenbach et al., 2001)); ADHD 195 symptoms (YSR-DSM-Oriented subscale, T-score $\geq 65$; Achenbach et al., 2001; Achenbach \& 196 Rescorla, 2001) ODD (YSR-DSM-Oriented subscale, T-score $\geq 65$ ); conduct problems (YSR-

197 DSM-Oriented subscale, T-score $\geq 65$ ); and self-harm behaviors (HBICA subscale, above the 198 90th percentile; Wang, 2012)). Specifically, individuals were identified as either meeting criteria 199 for a psychological problem (1) or not (0).

200

201

202

203

204

205

206

207

208

209

210

211

212

213

214

215

216

\section{Statistical analysis}

To compare the characteristics of each MVPA frequency group to sociodemographic variables, psychological problems and self-harm behaviors, F-tests were used for the continuous variables, and chi-square tests were used for the categorical variables. The effect size was estimated by $\eta^{2}$ for ANOVA or $\varphi / v$ for chi-square tests. All tests and analyses were examined by gender.

To evaluate the associations of MVPA frequency with psychological problems and selfharm behavior, multiple logistic regression analyses of the effect of MVPA frequency on the risk of psychological problems and self-harm behavior, adjusted for age, gender, grade (grade 7-9 or 10-12), BMI, SES, and ST, were conducted first for the total sample and then stratified by gender using MVPA frequency as the independent variable and each psychological problem or selfharm behavior as the dependent variable. Odds ratio (ORs) and 95\% confidence intervals (CIs) were used as the measurement values of the associations between MVPA frequency and psychological problems; with no MVPA being used as the reference group. For the regression models, all exposure variables were fully observed. However, there were different missing data percentages for the outcomes (depression, 2.9\%; anxiety, 1.9\%; GEBS problems, 7.2\%; ADHD, 10.3\%; ODD, 8.8\%; conduct problems, $11.0 \%$; and self-harm behavior, $0.7 \%$ ). A value of $p<$

PeerJ reviewing PDF | (2019:11:42937:2:0:NEW 6 Feb 2020) 
2170.05 (two-tailed) was used for statistical significance across all analyses. All statistical analyses

218 were performed using the software of SPSS 19.0 and STATA 12.0.

\section{Results}

220 Characteristics of the participants and MVPA

221

The proportion of boys and girls in the sample was $50.7 \%$ and $49.3 \%$, respectively. The

222 mean age (SD) of all participants was 15.18 (1.90) years and more than fifty percent $(50.1 \%)$ of

223

224

225

226

227

228

229

230

231

232

233

234

235

236

237

238

239

240

241

242

all participants were in junior high school. Across all participants, the mean BMI (SD) was 19.61 (3.35), and the mean SES score (SD) was 6.13 (1.64). The Pearson $\chi^{2}$ results indicated that MVPA frequency was significantly associated with television time $(\mathrm{p}<0.001$ for both gender) and playing video/computer games or using the computer for noneducational purpose time ( $p=0.011$ for boys; $p<0.001$ for girls).

High school students' characteristics involving the three levels of MVPA (i.e., no, moderate frequency, high frequency) significantly differed in all sociodemographic variables and ST. The participant characteristics of each MVPA frequency (stratified by gender) are reported in Table 1. For boys, more than thirty percent (33.1\%) reported no MVPA in the last seven days, $42.0 \%$ reported moderate (i.e., one to three days) MVPA in a week, and 24.9\% reported high (i.e., more than three days) MVPA in a week. Sociodemographic and ST (both time spent watching television and playing video/computer games) for boys were significantly different $(p<.05)$ across all three levels of MVPA with effect sizes ranging from 0.01 to 0.32 .

As for girls, approximately 49\% reported having no MVPA in a week, 36.8\% reported moderate (i.e., one to three days) MVPA in a week, and 14.2\% reported high (more than three days) MVPA in a week. Sociodemographic variables and both ST types differed significantly ( $p$ $<0.001)$. The effect sizes for these differences ranged from 0.02 to 0.47 .

\section{Distribution of psychological problems and self-harm behavior}

The prevalence of psychological problems and self-harm behavior, stratified by gender and MVPA frequency, are reported in Table 2. 
For boys, the risk for self-harm behavior and the examined psychological problems, with

244 the exception of ODD $(p=0.141)$ and conduct problems $(p=0.631)$, differed across MVPA

245 levels $(p<0.05)$. The effect sizes ranged from 0.04 to 0.10 . For girls, the risk for the examined

246 psychological problems, with the exception of conduct problems $(p=0.122)$, differed across

247 MVPA levels $(p<0.05)$. The effect sizes for these differences ranged from 0.04 to 0.08 . No

248 significant difference in the risk prevalence of self-harm behavior was found across MVPA

249 levels $(p=0.674)$.

250

251

252

253

254

255

256

257

258

259

260

261

262

263

264

265

266

267

268

269

270

271

\section{Associations between MVPA, psychological problems and self-harm behavior}

The results of the adjusted ORs and the corresponding 95\% CIs of the logistic regression analyses for each psychological problem and self-harm behavior regarding MVPA in the total sample are reported in Table 3.

Engaging in high MVPA was associated with a lower risk of depression $(\mathrm{OR}=0.67,95 \%$ CI: 0.59-0.77), anxiety (OR =0.75, 95\% CI: 0.60-0.94), and ADHD (OR = 0.76, 95\% CI: 0.600.98) compared to the reference group (i.e., no MVPA). Moderate MVPA was associated with a lower risk of depression ( $\mathrm{OR}=0.86,95 \% \mathrm{CI}$ : 0.78-0.95), ADHD (OR $=0.76,95 \% \mathrm{CI}$ : 0.63 $0.91)$, and $\mathrm{ODD}(\mathrm{OR}=0.86,95 \% \mathrm{CI}: 0.74-0.99)$ compared to the reference group.

The detailed results of the adjusted ORs and the corresponding 95\% CIs of the logistic regression analyses for each psychological problem and self-harm behavior regarding MVPA volumes stratified by gender are reported in Table 4 .

For boys, high MVPA was associated with a lower risk of depression $(\mathrm{OR}=0.68,95 \%$ CI: $0.57-0.81)$ and anxiety $(\mathrm{OR}=0.71,95 \% \mathrm{CI}: 0.53-0.96)$ compared to the reference group. Moderate MVPA was associated with a lower risk of $\mathrm{ADHD}$ symptom $(\mathrm{OR}=0.73,95 \% \mathrm{CI}$ : 0.56-0.94) compared to the reference group. For girls, both high and moderate MVPA were associated with a lower risk of depression (moderate MVPA: OR =0.81, 95\% CI: 0.70-0.94; high MVPA: $\mathrm{OR}=0.67,95 \% \mathrm{CI}: 0.54-0.83$ ) compared to the reference group. High MVPA was associated with a lower risk of ADHD symptom $(\mathrm{OR}=0.58,95 \% \mathrm{CI}$ : 0.37-0.89) compared to the reference group. Only a moderate MVPA was associated with a lower risk of ODD (OR = 0.79, 95\% CI: 0.64-0.97). No significant association was found between MVPA and self-harm behavior or other psychological problems for either gender. 


\section{Discussion}

273

274

275

276

277

278

279

280

281

282

283

284

285

286

287

288

289

290

291

292

293

294

295

296

297

298

299

300

301

The present study examined the potential benefits of MVPA on psychological health in Chinese adolescents. In this large population-based investigation, a higher frequency MVPA was associated with less depression regardless of gender. ADHD and ODD symptoms decreased based on gender and the frequency of MVPA. Importantly, the various associations of MVPA with anxiety, ADHD, and ODD in both boys and girls indicated that gender and frequency of MVPA plays a role in the association between MVPA and psychological problems. These results support recommendations from the WHO concerning MVPA in adolescents (WHO, 2010).

Participants engaging in no MVPA during the previous week were observed in $33.1 \%$ of boys and $49 \%$ of girls. It is worth noting that the present study's rates of no MVPA for females is higher than ratings reported in a previous study conducted by Sun and colleagues (2014), which measured PA using moderate PA (at least 20 min each day) or vigorous PA (at least 30 min each day). Moreover, Sun et al., (2014) revealed that $42.8 \%$ of girls reported no vigorous PA, and $40.6 \%$ of girls reported no moderate PA. This disparate finding is reasonable given that we used the WHO daily recommended amount of MVPA (60 min per day) for children and adolescents (WHO, 2010). Furthermore, we found that only $24.9 \%$ of boys and $14.2 \%$ of girls had a high frequency of MVPA, indicating that there is a low rate of high school students who have enough MVPA in mainland China. We also found a significant difference between the three different MVPA frequencies and age for these junior high school students. For both genders, younger students engaged in more MVPA. Furthermore, a significantly different prevalence of psychological problems based on the frequency of MVPA and gender was found. After adjustment, logistic regression analyses revealed different associations between individual psychological problems, self-harm behavior and MVPA for each gender. For male and female adolescents, the risk of depression decreased consistently with more MVPA. The effects of the frequency of MVPA are in line with the findings of previous studies (Ahn \& Fedewa, 2011; Annesi, 2005; Brown et al., 2013; Cao et al., 2011; Tao et al., 2007).

Surprisingly, in the present study, a high frequency of MVPA was associated with less anxiety in boys, but not in girls. The significant association found in boys in this study is similar to the findings in another Chinese study and international research (McDowell, MacDonncha, \& Herring, 2017; McMahon et al., 2017; Tajik et al., 2017; Wang et al., 2014). However, a lack of 
302 consensus remains given that the present study's results contradict studies conducted in an

303 eastern inner province of China (Cao et al., 2011; Tao et al., 2007), which suggested no

304 significant associations between PA and anxiety in high school students. These differences could

305 be explained by how previous studies defined PA, as well as participants' sociodemographic

306 factors. The participants in the aforementioned studies were recruited solely from one province

307 in China rather than across multiple provinces and centers, as was the case in the present study.

308 Thus, the present findings are more generalizable to all Chinese adolescents. More importantly,

309 these studies did not stratify the analysis of the associations by gender.

310 In the present study, we did not find significant associations between MVPA and anxiety

311 in girls, and only high MVPA was associated with less anxiety in the total sample. This result is

312 also different from the study by Wilkinson and colleagues (2017), which suggested that lower

313 social physique anxiety was associated with more PA per day for American and Mexican girls

314 and Mexican-born boys but not in American-born boys. However, for European adolescents, a

315 negative correlation was found between the frequency of PA and anxiety for both boys and girls

316 (McMahon et al., 2017). This discrepancy might be explained by a culture-specific effect. In

317 China, given that traditional masculine gender-role stereotyping is pervasive across society, this

318 may provide justification for boys to take part in PA. In this study, the higher prevalence of

319 MVPA (at least one day/week) for boys (66.9\%) compared to girls (51.0\%) also supports this

320 claim. This gender-role stereotyping may provide boys with more psychological rewards, which

321 in turn, may produce anti-anxiety effects (Brunes, Gudmundsdottir, \& Augestad, 2015; Salmon,

322 2001). On the other hand, in China, the traditional feminine gender-role stereotype dictates that

323 girls should be gentle and non-muscular. As a result, performing any form of PA may go against

324 cultural norms and evoke unpleasant feelings. In addition, in the study by McMahon et al.,

325 (2017), although a negative association was found between the frequency of PA and anxiety for

326 both genders, at the highest levels of PA, higher anxiety was associated with a greater frequency

327 of PA in girls. This finding leaves open the question of whether PA and anxiety differs across

328 gender, with the possibility of negative effects of PA on anxiety for girls. The existing evidence

329 regarding gender differences in the association between PA and anxiety is limited and as such,

330 more research is needed to clarify this issue.

331 This study is the first to examine the association between MVPA and ADHD, ODD, and

332 conduct problems in Chinese adolescents. For MVPA and ADHD, significant associations were 
333 found in both genders. These results are consistent with the finding by Nylander et al. (2017),

334 who found that ADHD was associated with low PA in adolescents with type I diabetes.

335 Interestingly, gender influences this association. For boys, moderate MVPA was associated with

336 fewer ADHD symptoms compared with no MVPA. On the other hand, only high MVPA was

337 associated with fewer ADHD symptoms in girls compared to no MVPA. Regarding ODD, only a

338 moderate frequency of MVPA was associated with fewer ODD symptoms in girls compared to

339 no MVPA. The mechanisms that influence these gender differences are complex and one

340 possibility is that they are influenced by cultural and social norms (Ahn et al., 2018).

341 Alternatively, these outcomes may reflect gender differences in coping mechanisms when

342 dealing with psychological problems (Nolen-Hoeksema, 1987). For example, Nolen-Hoeksema

343 (1987) noted that boys are more likely to engage in distracting behaviors such as PA, which in

344 turn, inhibits their negative moods while girls are more likely to amplify their moods with

345 ruminative responses about their state of discomfort. In turn, these approaches could lead to a

346 higher sensitivity of the therapeutic effects of PA in girls. The present study's results support this

347 hypothesis. Furthermore, given the masculine stereotyping and gender-roles within Chinese

348 society, MVPA behaviors are conceivably more appealing to Chinese male adolescents.

349 Therefore, it is probable that only a high frequency of MVPA may exert a therapeutic effect on

350 negative moods for Chinese male adolescents.

351 The present study focused on the frequency of MVPA for adolescents recommended by

352 the WHO (2010) and comprehensively considered multiple psychological problems experienced

353 by adolescents. The findings extend previous research in this field. The current study also adds

354 important insight into the potential role of gender on the relationship between MVPA and

355 depression, anxiety, ADHD, and ODD, which indicates that MVPA might be an effective role in

356 reducing psychological problems. This may provide evidence for the development of policies or

357 recommendations regarding appropriate levels of MVPA for juveniles in mainland China, and

358 potentially other countries or regions. Additionally, unlike previous Chinese studies, which are

359 not representative of the broader Chinese population, the recruitment method in the present study

360 enables a better generalizability of the results to all Chinese adolescents. This is because the

361 participants were recruited from major cities in North, South, East, West and Central China.

362 Notwithstanding the strengths, there are also some limitations to consider. First, this study

363 employs a cross-sectional observational design. Therefore, we cannot address the issue of 
364 causality of the associations between MVPA and psychological problems and self-harm behavior.

365 Second, although the data analyses were adjusted for potential confounding variables, it is not

366 possible to exclude the potential biases induced by other factors. For instance, the pressure of

367 academic performance may lead to less PA and greater anxiety at the same time. In addition,

368 self-reported MVPA and psychological problems and self-harm behavior may cause

369 measurement errors, which would subsequently influence the results. Finally, despite the better

370 generalizability of the results than that of previous Chinese studies, the present study did not

371 recruit participants from rural areas or other cities in China. Future Chinese studies should

372 endeavor to explore the effects of PA on psychological problems and self-harm behavior among

373 rural Chinese adolescents.

374

375 Conclusions

376 The present study provides empirical support for the recommendations put forth by the

377 WHO. Additionally, this study contributes to the body of literature by informing researchers,

378 health practitioners and policymakers of the psychological health benefits brought upon by PA.

379 Overall, for mainland Chinese high school students, the level of MVPA determined by the WHO

380 was associated with a lower risk of depression, anxiety, ADHD, and ODD. These associations

381 were influenced by gender and the frequency of MVPA. Ultimately, the results indicate that PA

382 interventions targeting specific psychological issues require understanding of how gender and

383 frequency of MVPA may influence the therapeutic effects amongst Chinese adolescents.

386 Acknowledgements

387

The authors would like to acknowledge the help of the following individuals for contributing to participants recruitment and measurement at the study centers: Wenbing Gao (Chinese Academy of Sciences), Wei Hong and Jing Liu (Peking University), Zhengyan Jiang

390 (Zhejiang University), Yanqing Tang (China Medical University), Jin Jing (Sun Yat-Sen

391 University), Wenqing Fu (Suzhou University), Yi Huang (Sichuan University), and Jianqun Fang

392 (Ningxia Medical University). And the first author's work was helped by China Scholarship

393 Council. 


\section{References}

396 Achenbach, T. M., Dumenci, L., \& Rescorla, L. A. (2001). Ratings of relations between DSM-

397

398

399

400

401

402

403

404

405

406

407

408

409

410

411

412

413

414

415

416

417

418

419

420

421

422

423

424

425

IV diagnostic categories and items of the CBCL/6-18, TRF, and YSR. Burlington,VT: University of Vermont

Achenbach, T. M., \& Rescorla, L. (2001). Manual for the ASEBA school-age forms \& profiles: An integrated system of multi-informant assessment. Burlington. VT: Aseba

Ahn, J. V., Sera, F., Cummins, S., \& Flouri, E. (2018). Associations between objectively measured physical activity and later mental health outcomes in children: findings from the UK Millennium Cohort Study. Journal of Epidemiology and Community Health, 72(2), 94-100. doi: 10.1136/jech-2017-209455

Ahn, S., \& Fedewa, A. L. (2011). A Meta-analysis of the Relationship Between Children's Physical Activity and Mental Health. Journal of Pediatric Psychology, 36(4), 385-397. doi: 10.1093/jpepsy/jsq107

Annesi, J. J. (2005). Correlations of Depression and Total Mood Disturbance with Physical Activity and Self-Concept in Preadolescents Enrolled in an After-School Exercise Program 1. Psychological Reports., 96(3c), 891-898.

Brener, N. D., Kann, L., McManus, T., Kinchen, S. A., Sundberg, E. C., \& Ross, J. G. (2002). Reliability of the 1999 youth risk behavior survey questionnaire. Journal of Adolescent Health, 31(4), 336-342.

Brown, H. E., Pearson, N., Braithwaite, R. E., Brown, W. J., \& Biddle, S. J. (2013). Physical activity interventions and depression in children and adolescents. Sports Medicine 43(3), 195-206.

Brunes, A., Gudmundsdottir, S. L., \& Augestad, L. B. (2015). Gender-specific associations between leisure-time physical activity and symptoms of anxiety: the HUNT study. Social Psychiatry \& Psychiatric Epidemiology, 50(3), 419-427.

Buffart, L. M., Singh, A. S., van Loon, E. C., Vermeulen, H. I., Brug, J., \& Chinapaw, M. J. (2014). Physical activity and the risk of developing lung cancer among smokers: A metaanalysis. Journal of Science and Medicine in Sport, 17(1), 67-71.

Cao, H., Qian, Q., Weng, T., Yuan, C., Sun, Y., Wang, H., \& Tao, F. (2011). Screen time, physical activity and mental health among urban adolescents in China. Preventive Medicine, 53(4-5), 316-320. doi: 10.1016/j.ypmed.2011.09.002 
426 Carli, V., Hoven, C. W., Wasserman, C., Chiesa, F., Guffanti, G., Sarchiapone, M., .. .

427

428

429

430

431

432

433

434

435

436

437

438

439

440

441

442

443

444

445

446

447

448

449

450

451

452

453

454

455

456

Wasserman, D. (2014). A newly identified group of adolescents at "invisible" risk for psychopathology and suicidal behavior: findings from the SEYLE study. World Psychiatry, 13(1), 78-86. doi: 10.1002/wps.20088

Daley, A. J., Copeland, R. J., Wright, N. P., Roalfe, A., \& Wales, J. K. (2006). Exercise therapy as a treatment for psychopathologic conditions in obese and morbidly obese adolescents: a randomized, controlled trial. Pediatrics, 118(5), 2126-2134.

Gapin, J. I., Labban, J. D., Etnier, J. L., \& Donnelly, J. E. (2011). The effects of physical activity on attention deficit hyperactivity disorder symptoms: the evidence. Preventive Medicine, 52(52 Suppl 1), S70-S74.

Gore, F. M., Bloem, P. J., Patton, G. C., Ferguson, J., Joseph, V., Coffey, C., . . Mathers, C. D. (2011). Global burden of disease in young people aged 10-24 years: a systematic analysis. Lancet, 377(9783), 2093-2102.

Larun, L., Nordheim, L., Ekeland, E., Hagen, K., \& Heian, F. (2006). Exercise in prevention and treatment of anxiety and de-pression among children and young people. Cochrane Database of Systematic Reviews, 4.

Liu, M., Ming, Q., Yi, J., Wang, X., \& Yao, S. (2016). Screen Time on School Days and Risks for Psychiatric Symptoms and Self-Harm in Mainland Chinese Adolescents. Frontiers in Psychology, 7(502).

Liu, M., Wu, L., \& Yao, S. (2016). Dose-response association of screen time-based sedentary behaviour in children and adolescents and depression: a meta-analysis of observational studies. British Journal of Sports Medicine, 50(20), 1252-1258.

March, J. S., Parker, J. D., Sullivan, K., Stallings, P., \& Conners, C. K. (1997). The Multidimensional Anxiety Scale for Children (MASC): factor structure, reliability, and validity. Journal of the American Academy of Child and Adolescent Psychiatry, 36(4), 554-565.

McDowell, C. P., MacDonncha, C., \& Herring, M. P. (2017). Brief report: Associations of physical activity with anxiety and depression symptoms and status among adolescents. Journal of Adolescence, 55, 1-4. doi: 10.1016/j.adolescence.2016.12.004

McMahon, E. M., Corcoran, P., O'Regan, G., Keeley, H., Cannon, M., Carli, V., ... Wasserman, D. (2017). Physical activity in European adolescents and associations with anxiety, 
457

458

459

460

461

462

463

464

465

466

467

468

469

470

471

472

473

474

475

476

477

478

479

480

481

482

483

484

485

486

487

depression and well-being. European Child \& Adolescent Psychiatry, 26(1), 111-122. doi: 10.1007/s00787-016-0875-9

Merikangas, K. R., He, J. P., Burstein, M., Swanson, S. A., Avenevoli, S., Cui, L., . . Swendsen, J. (2010). Lifetime prevalence of mental disorders in U.S. adolescents: results from the National Comorbidity Survey Replication--Adolescent Supplement (NCS-A). J Am Acad Child Adolesc Psychiatry, 49(10), 980-989.

Mokdad, A. H., Forouzanfar, M. H., Daoud, F., Mokdad, A. A., El Bcheraoui, C., Moradi-Lakeh, M., ... \& Kravitz, H. (2016). Global burden of diseases, injuries, and risk factors for young people's health during 1990-2013: a systematic analysis for the Global Burden of Disease Study 2013. Lancet, 387(10036), 2383-2401.

Nolen-Hoeksema, S. (1987). Sex differences in unipolar depression: evidence and theory. Psychological bulletin, 101(2), 259-282.

Nylander, C., Tindberg, Y., Haas, J., Swenne, I., Torbjörnsdotter, T., Akesson, K., . . Fernell, E. (2018). Self- and parent-reported executive problems in adolescents with type 1 diabetes are associated with poor metabolic control and low physical activity. Pediatric diabetes, 19(1), 98-105.

World Health Organization.. (2010). Global Recommendations on Physical Activity for Health. World Health Organization.

Patton, G. C., Coffey, C., Cappa, C., Currie, D., Riley, L., Gore, F., . . Sangowawa, A. O. (2012). Health of the world's adolescents: a synthesis of internationally comparable data. Lancet, 379(9826), 1665-1675.

Primack, B. A., Swanier, B., Georgiopoulos, A. M., Land, S. R., \& Fine, M. J. (2009). Association between media use in adolescence and depression in young adulthood: a longitudinal study. Archives of General Psychiatry, 66(2), 181-188.

Radloff, L. S. (1991). The use of the Center for Epidemiologic Studies Depression Scale in adolescents and young adults. Journal of youth and adolescence, 20(2), 149-166.

Roberts, R. E., Lewinsohn, P. M., \& Seeley, J. R. (1991). Screening for adolescent depression: a comparison of depression scales. Journal of the American Academy of Child and Adolescent Psychiatry, 30(1), 58-66.

Salmon, P. (2001). Effects of physical exercise on anxiety, depression, and sensitivity to stress: A unifying theory. Clinical Psychology Review, 21(1), 33-61. 
488

489

490

491

492

493

494

495

496

497

498

499

500

501

502

503

504

505

506

507

508

509

510

511

512

513

514

515

516

517

Sawyer, S. M., Afifi, R. A., Bearinger, L. H., Blakemore, S.-J., Dick, B., Ezeh, A. C., \& Patton, G. C. (2012). Adolescence: a foundation for future health. Lancet, 379(9826), 1630-1640.

Sun, Y., An, J., Wang, X., Zu, P., \& Tao, F.-B. (2014). Gender- and Puberty-Dependent Association Between Physical Activity and Depressive Symptoms: National Survey Among Chinese Adolescents. Journal of Physical Activity and Health., 11(7), 1430-1437. doi: 10.1123/jpah.2012-0366

Tajik, E., Abd Latif, L., Adznam, S. N., Awang, H., Yit Siew, C., \& Abu Bakar, A. S. (2017). A study on level of physical activity, depression, anxiety and stress symptoms among adolescents. Journal of Sports Medicine and Physical Fitness, 57(10), 1382-1387. doi: 10.23736/s0022-4707.16.06658-5

Tao, F. B., Xu, M. L., Kim, S. D., Sun, Y., Su, P. Y., \& Huang, K. (2007). Physical activity might not be the protective factor for health risk behaviours and psychopathological symptoms in adolescents. Journal of Paediatrics and Child Health., 43(11), 762-767. doi: 10.1111/j.1440-1754.2007.01217.x

Vancampfort, D., Hallgren, M., Firth, J., Rosenbaum, S., Schuch, F. B., Mugisha, J., .. . Stubbs, B. (2017). Physical activity and suicidal ideation: A systematic review and meta-analysis. Journal of Affective Disorders, 225, 438-448.

Wang, H., Fu, J., Lu, Q., Tao, F., \& Hao, J. (2014). Physical activity, Body Mass Index and mental health in Chinese adolescents: a population based study. Journal of sports medicine and physical fitness, 54(4), 518-525.

Wang, M. (2012). Development and psychometric properties of the health-risk behavior inventory for Chinese adolescents. BMC Medical Research Methodology, 12(1), 94-94.

Wang, M., Armour, C., Wu, Y., Ren, F., Zhu, X., \& Yao, S. (2013). Factor Structure of the CES$\mathrm{D}$ and Measurement Invariance Across Gender in Mainland Chinese Adolescents. Journal of Clinical Psychology, 69(9), 966-979. doi: 10.1002/jclp.21978

Wang, M., Yi, J., Cai, L., Hu, M., Zhu, X., Yao, S., \& Auerbach, R. P. (2012). Development and psychometric properties of the health-risk behavior inventory for Chinese adolescents. BMC Medical Research Methodology, 12. doi: 10.1186/1471-2288-12-94

Werneck, A. O., Collings, P. J., Barboza, L. L., Stubbs, B., \& Silva, D. R. (2019). Associations of sedentary behaviors and physical activity with social isolation in 100,839 school 
518

519

520

521

522

523

524

525

526

527

528

529

530

531

532

533

534

students: The Brazilian Scholar Health Survey. General Hospital Psychiatry, 59, 7-13. doi: 10.1016/j.genhosppsych.2019.04.010

Wilkinson, A. V., Miller, E. E., Koehly, L. M., Daniel, C. R., \& Forman, M. R. (2017). Correlates of Physical Activity Differ by Sex and Country of Birth Among MexicanHeritage Youth. Journal of Immigrant and Minority Health, 19(2), 246-253. doi: 10.1007/s 10903-016-0451-X

Xin, X., Ming, Q., Zhang, J., Wang, Y., Liu, M., \& Yao, S. (2016). Four Distinct Subgroups of Self-Injurious Behavior among Chinese Adolescents: Findings from a Latent Class Analysis. Plos One, 11(7), e0158609.

Yao, S., Zhang, C., Zhu, X., Jing, X., McWhinnie, C. M., \& Abela, J. R. (2009). Measuring adolescent psychopathology: Psychometric properties of the self-report Strengths and Difficulties Questionnaire in a sample of Chinese adolescents. Journal of Adolescent Health, 45(1), 55-62.

Yao, S., Zou, T., Zhu, X., Abela, J. R., Auerbach, R. P., \& Tong, X. (2007). Reliability and validity of the Chinese version of the Multidimensional Anxiety Scale for Children among Chinese secondary school students. Child Psychiatry and Human Development, $38(1), 1-16$. 


\section{Table $\mathbf{1}$ (on next page)}

Characteristics of participants by MVPA level ${ }^{\dagger}$

Abbreviations: MVPA, moderate to vigorous physical activity; BMI, body mass index; SES, subjective economic status; ES, effect size

${ }^{\dagger}$ Percentages do not always equal 100 because of rounding. Values may not always sum to sample size because of missing data. ${ }^{\ddagger} J u n i o r$ grade indicates $7^{\text {th }}-9^{\text {th }}$ grade " SES was considered as continuous variables (ranging from 1-the worst, to 10-the best). 


\section{1}

\section{Table 1. Characteristics of participants by MVPA level ${ }^{\dagger}$}

\begin{tabular}{|c|c|c|c|c|c|c|c|c|c|}
\hline \multirow[b]{2}{*}{ Variables } & \multirow[b]{2}{*}{$\begin{array}{l}\text { Total } \\
\text { (13349) }\end{array}$} & \multicolumn{4}{|c|}{ boys (days/week) (N, \%) } & \multicolumn{4}{|c|}{ girls (days/week) $(\mathrm{N}, \%)$} \\
\hline & & $\begin{array}{l}0 \\
(2236 ; 33.1 \%)\end{array}$ & $\begin{array}{l}\geq 1 \text { to } \leq 3 \\
(2842 ; 42.0 \%)\end{array}$ & $\begin{array}{l}>3 \\
(1687 ; 24.9 \%)\end{array}$ & $\mathbf{E S} ; \mathbf{p}$ & $\begin{array}{c}0 \\
(3224 ; 49.0 \%)\end{array}$ & $\begin{array}{c}\geq 1 \text { to } \leq 3 \\
(2425 ; 36.8 \%)\end{array}$ & $\begin{array}{l}>3 \\
(935 ; 14.2 \%)\end{array}$ & ES;p \\
\hline Age, mean (SD) & $15.18(1.90)$ & $15.78(1.82)$ & $15.06(1.86)$ & $14.58(1.75)$ & $0.06 ;<0.001$ & $15.91(1.76)$ & $14.54(1.83)$ & $14.20(1.59)$ & $0.15 ;<0.001$ \\
\hline Junior grade $\$, N(\%)$ & $6836(50.1)$ & $697(31.2)$ & $1549(54.5)$ & $1231(73.0)$ & $0.32 ;<0.001$ & $824(25.6)$ & $1611(66.4)$ & $789(84.4)$ & $0.47 ;<0.001$ \\
\hline BMI, mean (SD) & $19.61(3.35)$ & $20.24(3.69)$ & $20.00(3.63)$ & $19.95(3.66)$ & $0.01 ; 0.028$ & $19.48(2.83)$ & $18.87(3.00)$ & $18.52(2.77)$ & $0.02 ;<0.001$ \\
\hline \multicolumn{10}{|c|}{ Exposure levels of television ( hours/school day), N (\% } \\
\hline 0 & 7869 (58.6) & $1349(60.4)$ & $1394(49.2)$ & $836(49.6)$ & \multirow[t]{4}{*}{$0.09 ;<0.001$} & $2182(67.8)$ & $1454(60.0)$ & $594(63.6)$ & \multirow[t]{4}{*}{$0.06 ;<0.001$} \\
\hline$\leq 1$ & 3903 (29.1) & $617(27.6)$ & $1029(36.3)$ & $553(32.8)$ & & $742(23.1)$ & $710(29.3)$ & $230(24.6)$ & \\
\hline$>1$ to $\leq 2$ & $935(7.0)$ & $142(6.4)$ & $265(9.3)$ & $136(8.1)$ & & $161(5.0)$ & $166(6.9)$ & $61(6.5)$ & \\
\hline$>2$ & $710(5.3)$ & $124(5.6)$ & $148(5.2)$ & $161(9.5)$ & & $133(4.1)$ & $92(3.8)$ & $49(5.2)$ & \\
\hline 0 & $9290(70.2)$ & $1491(67.6)$ & $1834(65.7)$ & $1060(64.8)$ & \multirow{4}{*}{$0.04 ; 0.011$} & $2418(75.7)$ & 1705 (71.4) & 714 (77.9) & \multirow{4}{*}{$0.05 ;<0.001$} \\
\hline$\leq 1$ & $2440(18.4)$ & 407 (18.5) & $589(21.1)$ & $324(19.8)$ & & $513(16.1)$ & $473(19.8)$ & $120(13.1)$ & \\
\hline$>1$ to $\leq 2$ & $761(5.8)$ & $142(6.4)$ & $191(6.8)$ & $104(6.4)$ & & $135(4.2)$ & $137(5.7)$ & $47(5.1)$ & \\
\hline$>2$ & $736(5.4)$ & $164(7.4)$ & $178(6.4)$ & $149(9.1)$ & & $130(4.1)$ & $74(3.1)$ & $35(3.8)$ & \\
\hline
\end{tabular}

Abbreviations: MVPA, moderate to vigorous physical activity; BMI, body mass index; SES, subjective economic status; ES, effect size

$\dagger$ Percentages do not always equal 100 because of rounding. Values may not always sum to sample size because of missing data.

$\$$ Junior grade indicates $7^{\text {th }}-9^{\text {th }}$ grade

๑ SES was considered as continuous variables (ranging from 1-the worst, to 10-the best).

$$
2
$$




\section{Table 2 (on next page)}

Prevalence $(\%)^{\dagger}$ of psychological problems by MVPA levels

Abbreviations: MVPA, moderate to vigorous physical activity; GEBS, general emotion, behavior, and sociality; ADHD, Attention deficit/hyperactivity disorder; ODD, oppositional defiant disorder; ES, effect size

${ }^{\dagger}$ Percentages do not always equal 100 because of rounding. Values may not always sum to sample size because of missing data. ${ }^{\ddagger}$ Total prevalence of oppositional defiant problems was $11.1 \%$ for more than $3 \%$ samples' T score was between 64.5 to 65 (cutoff : t-score $\geq \mathbf{6 4 . 5 0}$ ). 
Table 2. Prevalence (\%) ${ }^{\dagger}$ of psychological problems by MVPA levels

\begin{tabular}{|c|c|c|c|c|c|c|c|c|c|}
\hline \multirow{2}{*}{ Variables } & \multirow{2}{*}{$\begin{array}{c}\text { Total } \\
\text { N (\%) }\end{array}$} & \multicolumn{4}{|c|}{ boys (days/week) N (\%) } & \multicolumn{4}{|c|}{ girls (days/week) N (\%) } \\
\hline & & $\mathbf{0}$ & $\geq 1$ to $\leq 3$ & $>\mathbf{3}$ & $\mathbf{E S} ; \mathbf{p}$ & $\mathbf{0}$ & $\geq 1$ to $\leq 3$ & $>\mathbf{3}$ & $\mathbf{E S} ; \mathbf{p}$ \\
\hline Depression, N (\%) & $3539(27.3)$ & $729(34.3)$ & $779(28.5)$ & $369(22.7)$ & $0.10 ;<0.001$ & $924(29.1)$ & $558(23.3)$ & $180(19.6)$ & $0.08 ;<0.001$ \\
\hline Anxiety, N (\%) & $943(7.2)$ & $181(8.8)$ & $199(7.5)$ & $89(5.7)$ & $0.05 ; 0.002$ & $258(8.3)$ & $164(6.9)$ & $52(5.7)$ & $0.04 ; 0.019$ \\
\hline GEBS problems, N (\%) & $1264(10.2)$ & $244(12.3)$ & $241(9.1)$ & $139(8.8)$ & $0.05 ;<0.001$ & $351(11.7)$ & $216(9.5)$ & $73(8.0)$ & $0.05 ; 0.001$ \\
\hline ADHD, N (\%) & $766(6.4)$ & $151(7.9)$ & $142(5.6)$ & $93(6.2)$ & $0.04 ; 0.006$ & $225(7.7)$ & $121(5.5)$ & $34(3.9)$ & $0.06 ;<0.001$ \\
\hline ODD, N (\%) & $1352(11.1)$ & $236(12.1)$ & $273(10.4)$ & $186(12.0)$ & $0.03 ; 0.141$ & $355(12.0)$ & $212(9.4)$ & $90(10.0)$ & $0.04 ; 0.007$ \\
\hline Conduct problems, N (\%) & $867(7.3)$ & $137(7.3)$ & $169(6.7)$ & $97(6.5)$ & $0.01 ; 0.631$ & $232(8.0)$ & $179(8.2)$ & $53(6.1)$ & $0.03 ; 0.122$ \\
\hline Self-harm behavior, N (\%) & $1431(10.8)$ & $280(12.7)$ & $285(10.2)$ & $167(10.1)$ & $0.04 ; 0.007$ & $340(10.6)$ & $266(11.1)$ & $93(10.1)$ & $0.01 ; 0.674$ \\
\hline
\end{tabular}

Abbreviations: MVPA, moderate to vigorous physical activity; GEBS, general emotion, behavior, and sociality; ADHD, Attention deficit/hyperactivity disorder; ODD, oppositional defiant disorder; ES, effect size

$\dagger$ Percentages do not always equal 100 because of rounding. Values may not always sum to sample size because of missing data.

$\$$ Total prevalence of oppositional defiant problems was $11.1 \%$ for more than $3 \%$ samples' $\mathrm{T}$ score was between 64.5 to 65 (cutoff : t-score $\geq \mathbf{6 4 . 5 0}$ ). 


\section{Table 3 (on next page)}

Logistic regression analysis of MVPA on the risk of psychological problems and self-harm behavior in the total sample

Abbreviations: MVPA, moderate to vigorous physical activity; GEBS, general emotion, behavior, and sociality; ADHD, Attention deficit/hyperactivity disorder; ODD, oppositional defiant disorder; OR, adjusted odds ratio; $\mathrm{Cl}$, confidence interval

Adjusted variables: age, gender, junior grade, body mass index, subjective economic status, volume of television and video games/computer use for non-education purpose 
Table 3. Logistic regression analysis of MVPA on the risk of psychological problems and self-harm behavior in the total sample

\begin{tabular}{lcc}
\hline & \multicolumn{1}{c}{ Self-reported MVPA levels (days/week) } \\
\cline { 2 - 3 } Variables & \multicolumn{1}{c}{$\mathbf{1}$ to $\leq \mathbf{3}$} & $>\mathbf{3}$ \\
\hline Depression, OR $(95 \% \mathrm{CI}), \mathrm{p}$ & $0.86(0.78$ to 0.95$), 0.003$ & $0.67(0.59$ to 0.77$),<0.001$ \\
Anxiety, OR $(95 \% \mathrm{CI}), \mathrm{p}$ & $0.94(0.80$ to 1.11$), 0.447$ & $0.75(0.60$ to 0.94$), 0.014$ \\
GEBS problems, OR $(95 \% \mathrm{CI}), \mathrm{p}$ & $0.90(0.78$ to 1.04$), 0.157$ & $0.86(0.70$ to 1.04$), 0.121$ \\
ADHD, OR $(95 \% \mathrm{CI}), \mathrm{p}$ & $0.76(0.63$ to 0.91$), 0.004$ & $0.76(0.60$ to 0.98$), 0.031$ \\
ODD, OR $(95 \% \mathrm{CI}), \mathrm{p}$ & $0.86(0.74$ to 0.99$), 0.041$ & $0.99(0.83$ to 1.19$), 0.922$ \\
Conduct problems, OR $(95 \% \mathrm{CI}), \mathrm{p}$ & $1.03(0.86$ to 1.23$), 0.748$ & $0.84(0.66$ to 1.06$), 0.145$ \\
Self-harm behavior, OR $(95 \% \mathrm{CI}), \mathrm{p}$ & $0.96(0.84$ to 1.11$), 0.613$ & $0.85(0.77$ to 1.11$), 0.417$ \\
\hline
\end{tabular}

Abbreviations: MVPA, moderate to vigorous physical activity; GEBS, general emotion, behavior, and sociality; ADHD, Attention deficit/hyperactivity disorder; ODD, oppositional defiant disorder; OR, adjusted odds ratio; CI, confidence interval

Adjusted variables: age, gender, junior grade, body mass index, subjective economic status, volume of television and video games/computer use for non-education purpose 


\section{Table 4(on next page)}

Logistic regression analysis of associations of psychological problems and self-harm behavior with MVPA by gender

Abbreviations: MVPA, moderate to vigorous physical activity; GEBS, general emotion, behavior, and sociality; ADHD, Attention deficit/hyperactivity disorder; ODD, oppositional defiant disorder; OR, adjusted odds ratio; $\mathrm{Cl}$, confidence interval

Adjusted variables: age, junior grade, body mass index, subjective economic status, volume of television and video games/computer use for non-education purpose, Gender-matched no MVPA groups served as reference groups 
Table 4. Logistic regression analysis of associations of psychological problems and self-harm behavior with MVPA by gender

\begin{tabular}{|c|c|c|c|c|}
\hline \multirow{3}{*}{ Variables } & \multicolumn{4}{|c|}{ Self-reported MVPA levels (days/week) } \\
\hline & \multicolumn{2}{|c|}{ boys $(N=5651)$} & \multicolumn{2}{|c|}{$\operatorname{girls}(\mathrm{N}=\mathbf{5 5 1 2})$} \\
\hline & $\geq 1$ to $\leq 3$ & $>3$ & $\geq 1$ to $\leq 3$ & $>\mathbf{3}$ \\
\hline Depression, OR $(95 \% \mathrm{CI}), \mathrm{p}$ & 0.88 (0.77 to 1.01$), 0.068$ & $0.68(0.57$ to 0.81$),<0.001$ & $0.81(0.70$ to 0.94$), 0.005$ & $0.67(0.54$ to 0.83$),<0.001$ \\
\hline Anxiety, OR $(95 \% \mathrm{CI}), \mathrm{p}$ & $0.94(0.75$ to 1.18$), 0.604$ & $0.71(0.53$ to 0.96$), 0.024$ & $0.94(0.74$ to 1.19$), 0.588$ & $0.85(0.59$ to 1.21$), 0.358$ \\
\hline GEBS problems, OR $(95 \% \mathrm{CI}), \mathrm{p}$ & 0.84 ( 0.68 to 1.04$), 0.1034$ & $0.85(0.66$ to 1.10$), 0.211$ & $0.96(0.78$ to 1.19$), 0.702$ & $0.87(0.64$ to 1.20$), 0.400$ \\
\hline ADHD, OR $(95 \% \mathrm{CI}), \mathrm{p}$ & 0.73 (0.56 to 0.94$), 0.016$ & 0.88 (0.65 to 1.20$), 0.427$ & $0.81(0.62$ to 1.06$), 0.125$ & 0.58 ( 0.37 to 0.89$), 0.014$ \\
\hline ODD, OR $(95 \% \mathrm{CI}), \mathrm{p}$ & 0.92 (0.75 to 1.12$), 0.392$ & 1.12 (0.89 to 1.42$), 0.330$ & 0.79 (0.64 to .97$), 0.025$ & $0.84(0.63$ to 1.12$), 0.240$ \\
\hline Conduct problems, OR $(95 \% \mathrm{CI}), \mathrm{p}$ & $1.00(0.77$ to 1.30$), 0.995$ & 0.92 (0.67 to 1.26$), 0.595$ & $1.04(0.82$ to 1.33$), 0.734$ & $0.74(0.51$ to 1.06$), 0.100$ \\
\hline Self harm, OR $(95 \% \mathrm{CI}), \mathrm{p}$ & $0.87(0.72$ to 1.06$), 0.176$ & $0.92(0.72$ to 1.16$), 0.456$ & 1.01 ( 0.83 to 1.24$), 0.892$ & $0.92(0.69$ to 1.23$), 0.589$ \\
\hline
\end{tabular}

Abbreviations: MVPA, moderate to vigorous physical activity; GEBS, general emotion, behavior, and sociality; ADHD, Attention deficit/hyperactivity disorder; ODD, oppositional defiant disorder; OR, adjusted odds ratio; $\mathrm{CI}$, confidence interval

Adjusted variables: age, junior grade, body mass index, subjective economic status, volume of television and video games/computer use for non-education purpose,

Gender-matched no MVPA groups served as reference groups 